\title{
A colecção clássica de Lenoir \& Forster e o ensino de Paleontologia e Antropologia na Faculdade de Filosofia da Universidade de Coimbra
}

\author{
Pedro Callapez ${ }^{1,2}$; Maria Augusta Rocha ${ }^{3}$; Daniela Abrantes ${ }^{1}$; Ana Santos ${ }^{3}$; \\ Ricardo Paredes ${ }^{4}$; Júlio Marques ${ }^{1}$ \\ ${ }^{1}$ Departamento de Ciências da Terra / Museu Mineralógico e Geológico da Univ. Coimbra, PORTUGAL. \\ ${ }^{2}$ Centro de Geofísica da Univ. Coimbra, PORTUGAL. E-mail: callapez@dct.uc.pt. \\ ${ }^{3}$ Departamento de Ciência da Vida e CIAS - Centro de Investigação em Antropologia e Saúde da Univ. \\ Coimbra, PORTUGAL. \\ ${ }^{4}$ IMAR - CMA. Instituto do Mar, Universidade de Coimbra, PORTUGAL.
}

Palavras-chave: Lenoir \& Forster, Colecção de fósseis, Antropologia e Paleontologia, Universidade de Coimbra

\section{Resumo}

Após a reestruturação da Faculdade de Filosofia da Universidade de Coimbra, em 1885, procedeu-se à compra de numerosas colecções e modelos didácticos, com vista a apetrechar o ensino prático e experimental das novas cadeiras. Neste contexto, o papel desempenhado pelo Prof. Bernardino Machado foi assaz relevante, ao ocupar-se da Secção de Antropologia e da nova disciplina da área. Entre as colecções didácticas então adquiridas a comptoirs europeus, damos conta neste estudo, da de Lenoir \& Forster de Viena de Áustria, composta por 543 caixas com fósseis estratigráficos usados no ensino da história da Terra e da escala dos tempos geológicos. Esta colecção foi adquirida em 1892 e possui exemplares de excelente qualidade. É, também, bastante diversificada quanto a grupos taxonómicos, idades e jazidas, constituindo um excelente exemplo de um período em que muitos dos sítios clássicos europeus, hoje destruídos ou condicionados a recolhas, forneciam grande quantidade de fósseis para fins museológicos, educativos e de coleccionísmo.

Key-words: Lenoir \& Forster, Collection of fossils, Anthropology and Palaeontology, University of Coimbra

\footnotetext{
Abstract

After the reformation of the Faculty of Philosophy from the University of Coimbra, in 1885, were purchased many collections and didactic models to supply the practical and experimental teaching of the new disciplines, with new and more adequate materials. The role played by Prof. Bernardino Machado was very relevant within this context, when he occupied the head of the Section of Anthropology and the new discipline of the area. Among the didactic collections purchased just then to European comptoirs, the present
} 
study reveals that of Lenoir \& Forster from Vienna, which is a set of 543 boxes with stratigraphic fossils used in practical teaching of Earth history and geologic time scale. This collection purchased in 1892 has specimens of excellent quality and is highly diversified in taxonomic groups, ages and fossil sites. It is also an exceptional example of a historic period, when many of the classic sites of the European geological record that have been destroyed or restricted to field sampling in modern times, were yet fully accessible and yielded large numbers of fossil specimens to be used for museologic, educative or collectible purposes.

\section{Introdução}

As últimas décadas do século XIX significaram para Portugal um tempo de difícil adaptação à nova ordem imperialista europeia, alicerçada que estava na competição entre as grandes potências da época - Inglaterra, França e Alemanha - e num acentuado progresso científico e industrial, cujos ecos se faziam sentir de modo crescente nos meios políticos e académicos nacionais. Não obstante as primeiras reformas e tentativas de mitigar o atraso estrutural do país face a congéneres europeus remontem aos alvores do Fontismo, a sua progressão não será estranha às apreensões geradas pelo conflito com a Inglaterra e consequente risco de alienação de territórios ultramarinos da Coroa portuguesa, mas também face ao rearmamento crescente desses estados, que acabaria por culminar no hediondo conflito de 1914-18, para o qual o país se veria arrastado.

Perante um cenário político e socioeconómico desta natureza, a reformulação do ensino superior, politécnico, comercial e industrial, no sentido de o capacitar para a formação de mais e melhores quadros nas diversas áreas das ciências, com destaque para as aplicadas e de utilidade mais imediatista, sobreveio como uma necessidade perfeitamente natural e sentida. A reorganização de 1885 da Universidade de Coimbra enquadra-se nesta evolução de final de século, ao modernizar as antigas estruturas da Faculdade de Filosofia, algumas delas ainda arreigadas a resquícios de cunho pombalino. Não obstante, no dizer de Ferreira (1998), a Faculdade de Coimbra «assumiu exclusivamente o ensino das ciências básicas», num contexto europeu em que o ensino profissional se relegava para a competência de escolas profissionais.

Desta forma, ao ser modernizado o bacharelato em Filosofia Natural e autonomizadas as quatro secções do Museu de História Natural, com a criação de novas disciplinas mais consentâneas com as necessidades da época, a estruturação do ensino terá sido consideravelmente melhorada, permitindo a formação de quadros mais bem capacitados para a 
modernidade do conhecimento e derradeiros progressos das ciências naturais, antropológicas e arqueológicas do país. Recorde-se que, por esta data, já se perfaziam duas décadas de trabalho infatigável na III Comissão Geológica do Reino, sob a batuta de Carlos Ribeiro, Nery Delgado e diversos outros colaboradores de reconhecida competência (Antunes, 1986). A primeira carta geológica de Portugal havia sido impressa em 1876 e o reconhecimento estratigráfico do Reino, com as suas principais formações, fósseis e idades, já fora, em grande parte efectuado. Em simultâneo, os estudos de Arqueologia e de Antropologia pré-histórica eram uma prática corrente na década de 80 do século XIX, motivando questões polémicas e apaixonantes como a do homem do Terciário, a realização de congressos internacionais em território luso e a criação de salas de exposição com acervos consideráveis (Pires, 2009). Refira-se, como exemplo marcante, o IXe Congrès International d'Anthropologie \& d'Archéologie Préhistorique, em 1880.

Ao serem implementadas as novas cadeiras de Botânica, Zoologia, Mineralogia, Geologia e Antropologia, estas últimas, respectivamente, da responsabilidade dos professores Gonsalves Guimarães e Bernardino Machado, tornou-se necessário reapetrechar progressivamente os laboratórios práticos adstritos às respectivas secções, com recurso a novos equipamentos e colecções práticas que permitissem ao aluno uma experimentação mais efectiva dos ensinamentos teóricos em que as cátedras se fundamentavam. $\mathrm{Na}$ realidade, o manuseamento de espécimes e de modelos em colecções osteológicas, mineralógicas, litológicas ou paleontológicas com carácter didáctico, ainda hoje é uma prática indispensável para quem cursa o ensino liceal ou disciplinas básicas em cursos de ciências. Só desta forma é possível ao discente ultrapassar a abstracção inerente às imagens impressas nos manuais, ou expostas pelo professor no decurso da aula teórica, bem como diferentes concepções alternativas daí resultantes.

Após a reestruturação de 1885 e não olvidando a influência plausível de Bernardino Machado na tutela, a Faculdade de Filosofia promoveu a compra de diversas destas colecções didácticas a comptoirs europeus, naquilo que constituiu um enriquecimento considerável dos acervos de algumas secções do Museu de História Natural. Esta orientação terá continuado durante mais de duas décadas, até para além da reforma de 1911. No que concerne à Geologia, a dimensão destas colecções atinge algumas dezenas de milhar de espécimes e de modelos, repartidos entre amostras e lâminas delgadas de rochas, minerais, fósseis, modelos cristalográficos e modelos estratigráficos e tectónicos. Infelizmente, a sua utilização ao longo 
de várias décadas produziu desgastes consideráveis, perdendo-se ou deteriorando-se parte dos espécimes e rótulos originais. Este percurso rico em práticas lectivas, mas nem sempre revestido do cuidado que os acervos mereceriam, redundou numa imagem presente que não reflecte na sua plenitude o que teria sido a qualidade, ou mesmo, a sumptuosidade desses acervos. Não obstante, o fim primordial a que se destinavam terá sido alcançado, ao terem contribuído de modo significativo para a formação de várias gerações de bacharéis e de licenciados pela Universidade de Coimbra.

Nas páginas seguintes daremos notícia de uma destas colecções didácticas, adquirida ao comptoir Lenoir \& Forster de Viena de Áustria, uma das mais reputadas casas europeias do ramo na época (Figura 1). Para além da sua excelente qualidade, esta colecção sobressai dos restantes acervos contemporâneos do antigo Museu de História Natural por ser a mais bem conservada das de Paleontologia. Com efeito, dado ter sido adstrita à então Secção de Antropologia, não terá sido tão manipulada como as demais, permanecendo separada dos restantes acervos paleontológicos existentes na sua congénere de Mineralogia e Geologia. No seu todo, faculta-nos também uma visão preciosa do que seriam as colecções de âmbito escolar desta natureza comercializadas há mais de um século, quando as jazidas clássicas europeias ainda se encontravam relativamente intactas e acessíveis aos colectores da época.

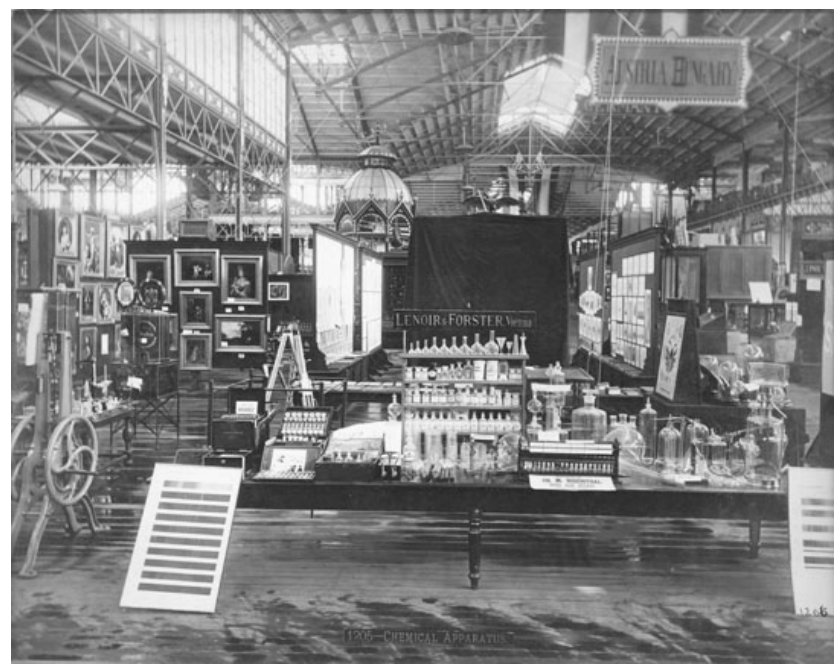

Figura 1. Stand do comptoir Lenoir \& Forster na Exposição de Filadélfia, no centenário da independência dos Estados Unidos (1876) [fonte: Print and Picture Collection, Free Library of Philadelphia]. 


\section{Aquisição e uso da colecção na Secção de Antropologia}

A colecção paleontológica com 543 espécies, animais e vegetais, adquirida a Lenoir \& Forster deu entrada no Gabinete do Museu da Universidade, em Janeiro de 1892. O processo de compra foi parcialmente reconstruído através das "Folhas de despesa" do Gabinete de Antropologia da Faculdade de Filosofia. Assim, em Dezembro de 1891 foi registado o valor de $2 \$ 205$ reis para pagamento do transporte e direitos a Henry Burnay \& $\mathrm{C}^{\mathrm{a}}$, empresa sediada em Lisboa. No mês seguinte é referida a despesa de $\$ 290$ reis a Valentim J. Rodrigues, de Coimbra, pelo transporte de dois caixotes com a colecção, desde a Estação do Caminho de Ferro desta cidade (Figura 2A). Apenas na "Folha de despesa" de Fevereiro surge a indicação da factura relativa à colecção no valor de 626 florins (Figura 2B). Ao câmbio corrente, correspondeu a 313\$000 reis, aos quais se acrescentaram $1 \$ 565$ com despesas de transferência. Dadas as alterações cambiais entre Fevereiro e Março de 1892 o custo efectivo em moeda Portuguesa sofreu novo acréscimo de $4 \$ 240$ reis. Contabilizadas as parcelas, o total dispendido na compra desta colecção foi de $321 \$ 350$ reis.

A aquisição desta colecção de fósseis integra-se num cariz de aulas teórico-práticas, tão caro a Bernardino Machado e lema do estudo de Filosofia Natural (Machado, 1904-05). Assim sendo, e complementando a preocupação constante de actualização e qualidade do ensino de Antropologia, foram adquiridos diversos materiais didácticos, como fósseis e instrumentos, entre outros (Rocha, 1995), de modo a munir o docente da $8^{a}$ cadeira do curso de Filosofia. Esta disciplina nasce a partir de dois projectos de lei apresentados pelo debutante deputado e professor Bernardino Machado, na sessão de 8 de Junho de 1883, na Câmara dos Deputados. Permitiu-se, desta forma, a criação da cadeira de Antropologia, paleontologia humana e archeologia prehistorica (Carta de Lei em 2 de Julho de 1885) na Faculdade de Filosofia da Universidade de Coimbra (Areia e Rocha, 1985).

Bernardino Luís Machado Guimarães (Figura 3) nasceu no Rio de Janeiro em 1851, tendo-se matriculado na Universidade de Coimbra em 1866, primeiro na Faculdade de Matemática e no ano seguinte na Faculdade de Filosofia. Da sua vasta carreira (Areia e Rocha, 1985; Rosa, 2006; Areia, 2007) referem-se alguns momentos académicos e políticos. Obteve o grau de doutor em Filosofia em 1876 e três anos depois foi nomeado lente catedrático da mesma Faculdade. Desde 1877 foi professor substituto, dando aulas de Física e de Agricultura Geral, Zootecnia, Economia Rural. Durante as legislaturas de 1890 a 1895 representou os estabelecimentos científicos superiores. Em 1898 presidiu também à Sociedade de Antropologia de 

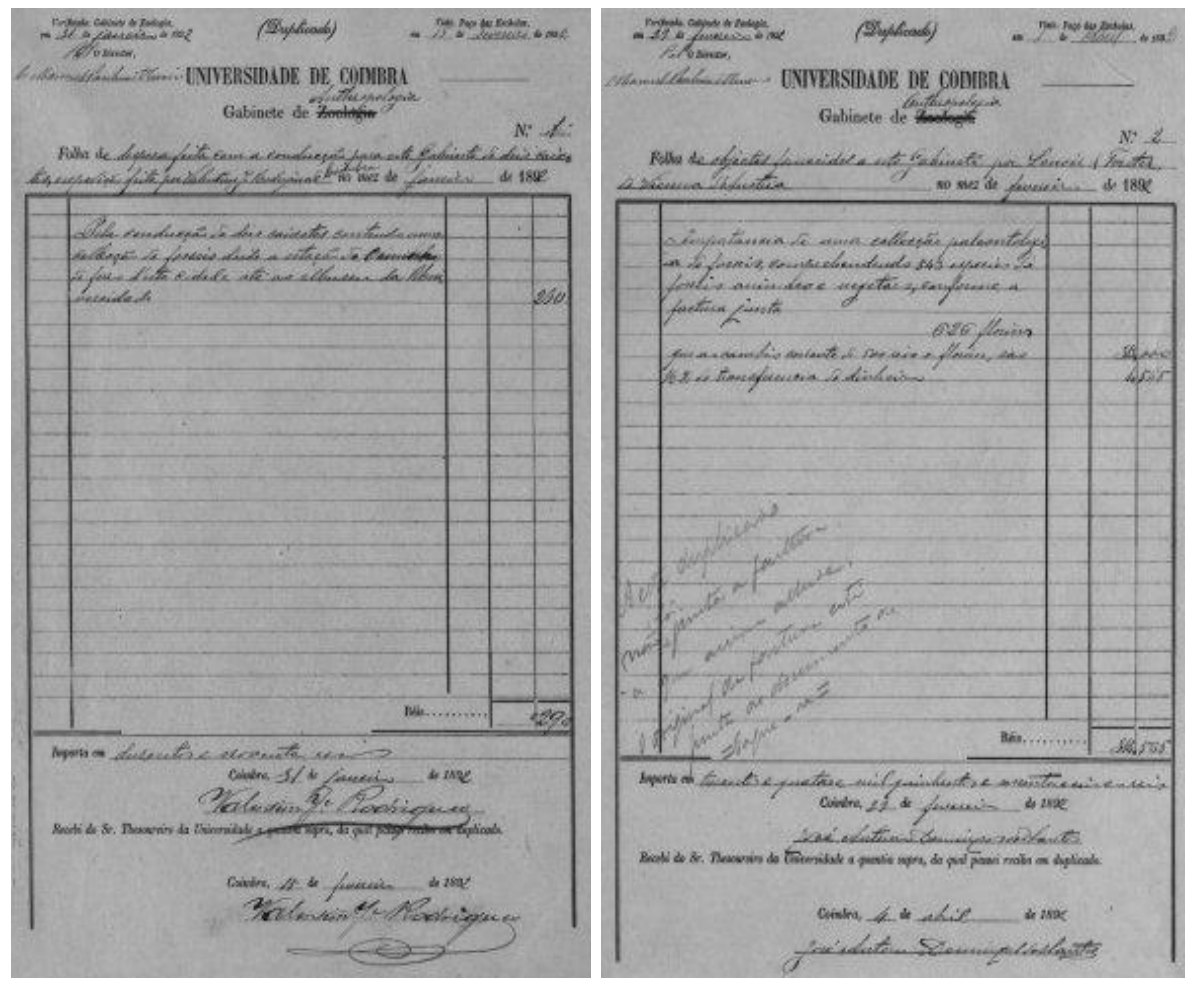

Figura 2. Folhas de despesa do Gabinete de Antropologia da Univ. Coimbra pelo transporte a Valentim J. Rodrigues (A) e pela colecção a Lenoir \& Forster (B). [fonte: Arquivo do Mus. Lab. Antropológico].

Coimbra. Em 1907 pediu a exoneração de professor da Universidade, publicada em 25 de Abril. Foi eleito Presidente da República entre 1915-17 e 1925-1926, tendo sido exilado após cada um dos mandatos. Em 1929 foi eleito para o Supremo Conselho do Grau 33 da Maçonaria. Tomou desde cedo posição pública contra a ditadura e viveu exilado em Espanha e França, até 1940. Faleceu com 93 anos no Porto e o funeral em realizou-se Vila Nova de Famalicão, sob grande repressão policial. Setenta anos após a sua exoneração da Universidade recebeu postumamente a Ordem da Liberdade. 


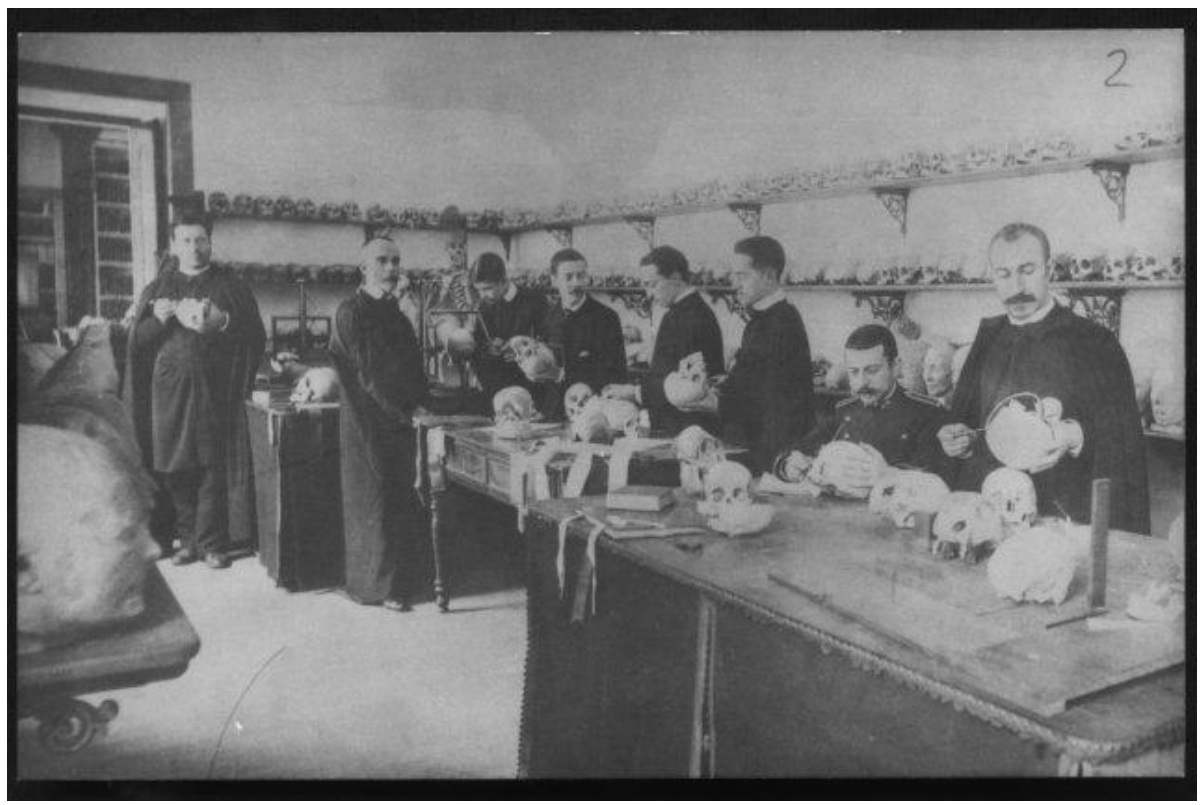

Figura 3. Bernardino Machado com os discípulos - Sidónio Pais (aluno fardado) - no curso de Antropologia de 1896-97 leccionado no Colégio de Jesus [fonte: Arquivo do Mus. Lab. Antropológico].

Apesar de Bernardino Machado ser o professor titular da cadeira, nem sempre a regeu (Figura 4), dada a sua intensa actividade nomeadamente como conselheiro e par do Reino (Areia e Rocha, 1985). O ensino desta nova disciplina criou um tal crédito que numerosos alunos de outras faculdades a frequentavam voluntariamente (Sueiro, 1944).

\section{Itinerários da colecção}

Esta colecção integrou durante 116 anos (de 1892 a 2008) os acervos que acompanharam a disciplina da Antropologia no périplo pelas suas diferentes instalações. De 1892 a 1911 permaneceu no Colégio de Jesus, onde estava instalada a Faculdade de Filosofia Natural; posteriormente, foi transferida para o Colégio de São Boaventura, após a reforma e a adaptação da sua bela arquitectura (Figuras 5A e B); em 1949, em consequência das obras da cidade universitária, mudou, provisoriamente, para o Colégio de S. Bento, mas o provisório tornou-se definitivo, e as instalações foram inauguradas oficialmente em Outubro de 1966 (Areia et al., 1991). Em 2008, foi estabelecido um protocolo em que o Museu Antropológico cedeu, a título de depósito, a colecção de Lenoir \& Forster ao Museu Mineralógico e 
Geológico. Apesar de tantas "residências" a colecção regressou bem preservada ao edifício onde chegou, em 1892, vinda de Viena de Áustria.

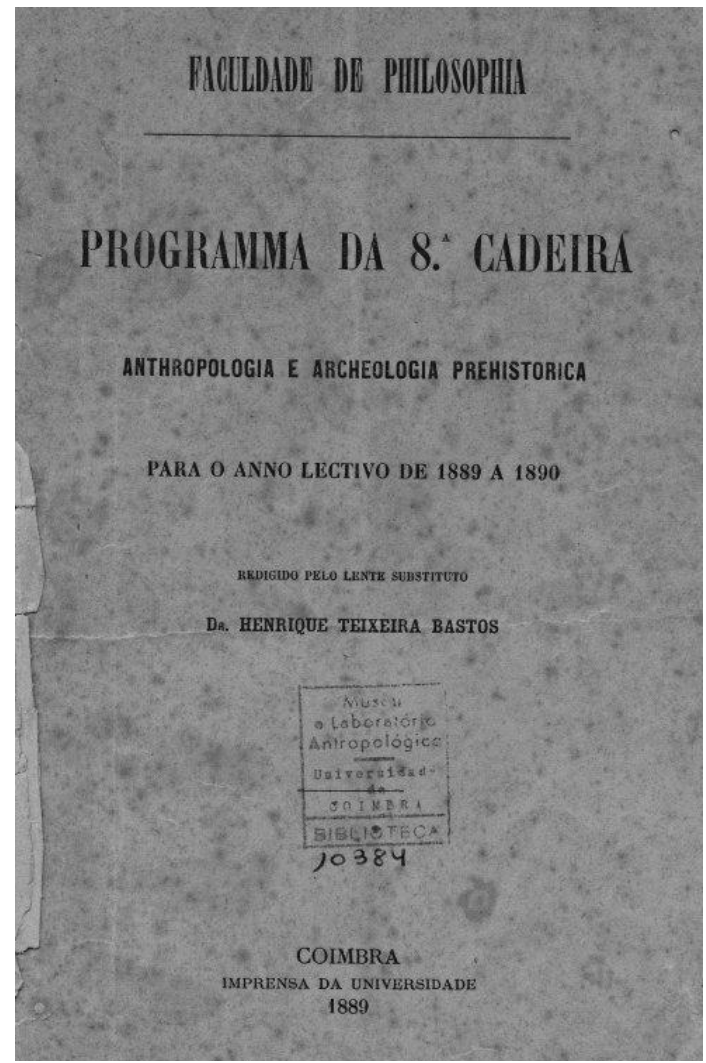

Figura 4. Frontispício do Programa da $8^{\mathrm{a}}$ cadeira da Faculdade de Filosofia (Bastos, 1889). [fonte: Arquivo do Mus. Lab. Antropológico].

\section{Conteúdo e significado}

A colecção de Lenoir \& Forster encontra-se exposta, ainda que em parte, numa das salas de exibição permanente do Museu Mineralógico e Geológico da Universidade de Coimbra, no primeiro piso do edifício do Colégio de Jesus, conservando-se o restante acervo na reserva e sendo tudo parte do espólio recém-cedido à Fundação do Museu da Ciência.

O inventário da colecção compreende 543 itens ordenados sequencialmente (Anexo 1), cada qual designando um ou mais espécimes numerados e conservados em caixas quadradas de polietileno transparente com revestimento de algodão colorido, acompanhadas do respectivo rótulo timbrado e manuscrito da casa de Viena de Áustria (Figura 6). Este acervo encontrava-se guardado, originalmente, em caixas de cartão azul, também 
elas conservadas em reserva. Dos 543 itens verifica-se a falta de 14, embora devam corresponder a alguns espécimes com numeração e rótulo extraviados, que acompanham os restantes.
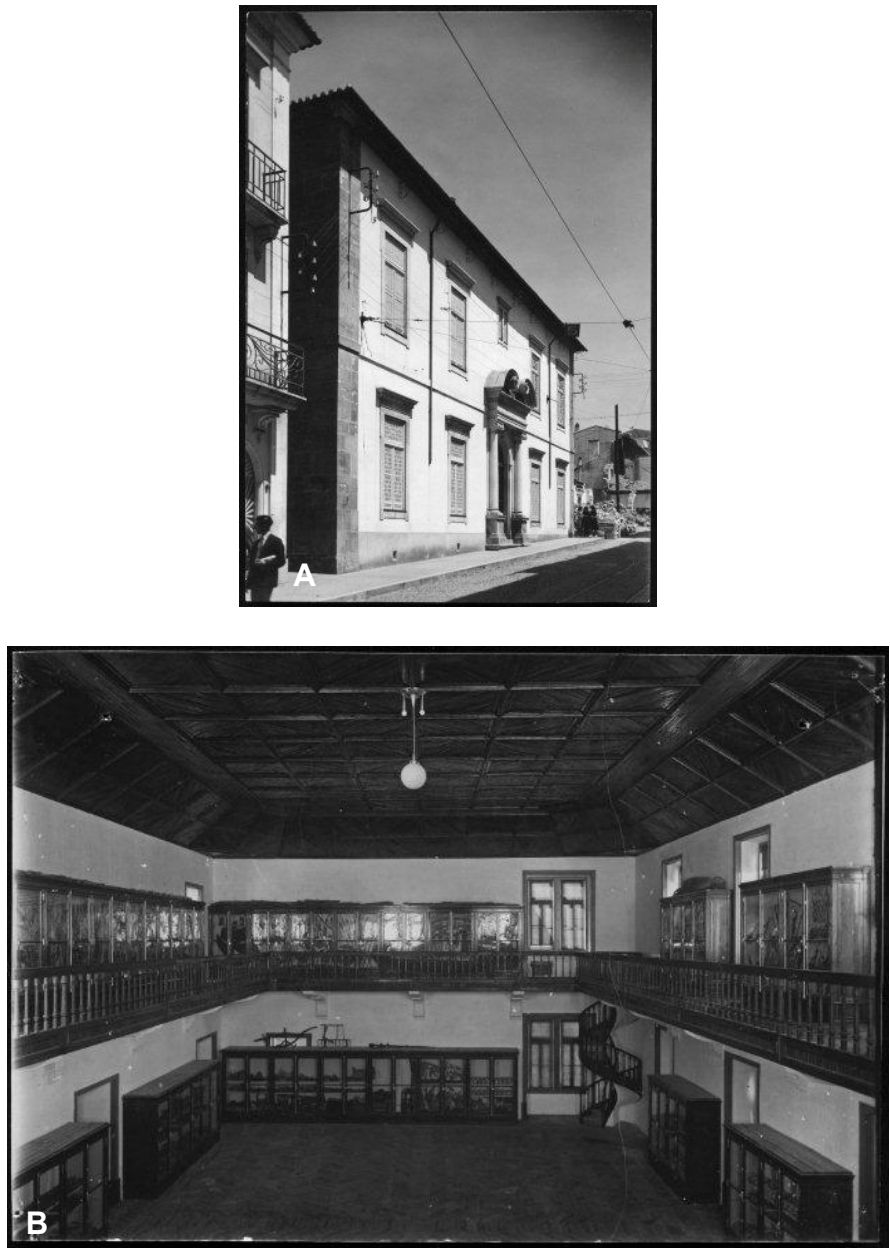

Figura 5. Colégio de S. Boaventura, fachada principal (A) voltada para a Rua Larga e Sala de Exposição de material (B). [fonte: Arquivo do Mus. Lab. Antropológico].

Em conjunto com a colecção de Lenoir \& Forster coexistem, também, diversos fósseis com rótulos do comptoirs Éloffe Boubée e Émile Deyrolle (fils) de Paris. Este facto realça que as aquisições da então Secção de Antropologia se terão também alargado a casas francesas, à semelhança do que se verificou na Secção de Mineralogia e Geologia e noutras instituições portuguesas contemporâneas, ligadas ao ensino prático das ciências. 
De entre vários aspectos que denotam a excelente qualidade da colecção e o elevado grau de profissionalismo e de conhecimento subjacente à sua preparação, destacamos o cuidado tido no preenchimento dos rótulos quanto à sistematização e taxonomia dos fósseis, classificados até à espécie salvo raras excepções e acompanhados do nome abreviado do Autor, ao bom gosto da época. O mesmo sucede com as indicações de jazida e de localidade, assim como com o posicionamento estratigráfico, embora a caligrafia manuscrita nem sempre seja de fácil leitura. É interessante notar que muitas destas jazidas e localidades correspondem a sítios clássicos da Geologia europeia, norte-americana e norte-africana, em muitos casos inacessíveis ou fortemente condicionados a recolhas actuais, facto que contribui para incrementar ainda mais a relevância do conjunto.
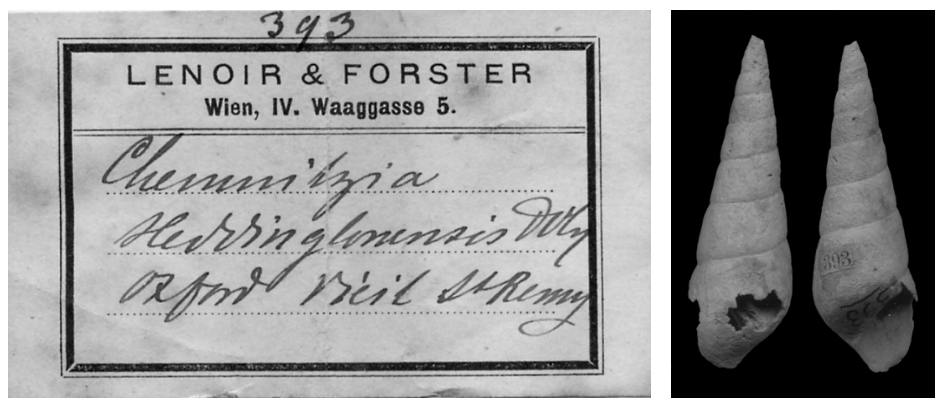

Figura 6. Rótulo e respectivo espécime da colecção didáctica de Paleontologia adquirida ao comptoir Lenoir \& Forster de Viena de Áustria [Fonte: reserva do Mus. Miner. Geológico].

Uma apreciação da qualidade dos exemplares, tendo em conta os fins a que se destinava este tipo de colecções - de coleccionísmo, científico e, sobretudo, didáctico - sugere que terá existido uma preocupação constante em evitar a inserção de moldes e de espécimes deficientemente preservados, em detrimento de conchas, tecas e outras partes esqueléticas ornamentadas que caracterizassem adequadamente os grupos taxonómicos maiores, bem como os géneros e espécies em causa. Numa acepção de coleccionísmo, a maioria destes exemplares podem ser considerados como de âmbito comum. No entanto, e muito possivelmente, como parte de estratégia adoptada para tornar as colecções mais vendáveis e apetecíveis a instituições universitárias e a coleccionadores particulares, foram adicionados vários espécimes de grande qualidade, com destaque para crustáceos, peixes e outros vertebrados meso-cenozóicos. Esta estratégia ainda hoje se aplica em colecções modernas: importa mostrar ao aluno uma panorâmica das formas mais comuns e representativas do registo fóssil, mas com recurso à inclusão de 
exemplares raros ou de aparência espectacular, para que este se motive e se sensibilize para a Paleontologia.

O carácter didáctico mais do que evidente e a consequente organização da colecção nesse sentido, estão bem patentes na diversidade taxonómica, temporal e geográfica do conjunto. Nesse sentido, foram representados quase todos os principais grupos de macrofósseis característicos ou de fácies do Fanerozóico, numa proporção que se aproxima da sua abundância relativa no registo fóssil (Figura 7). É interessante notar, todavia, a inexistência de exemplares de graptolites, um dos mais importantes grupos das paleofaunas do Paleozóico inferior, assim como a escassez de amostras com microfósseis e icnofósseis.

A numeração original da colecção também segue o ordenamento sistemático dos diferentes grupos representados. Tem início com fósseis vegetais, segue um modelo de organização dos principais filos de invertebrados, no sentido de uma complexidade anatómica crescente e termina com amostras de protocordados e de vertebrados.

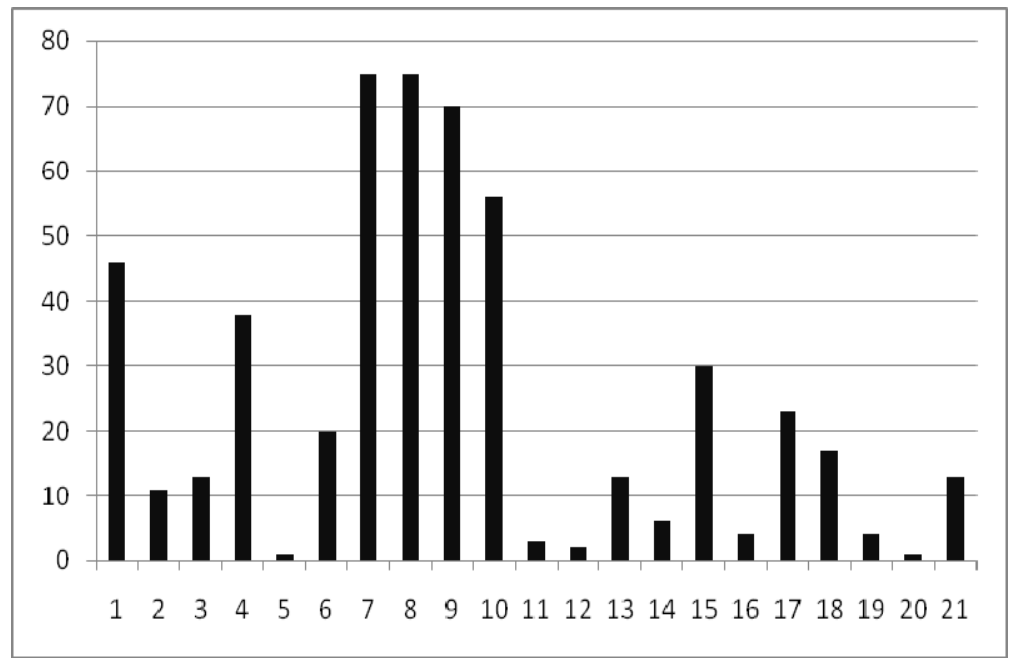

Figura 7. Abundância relativa dos grupos taxonómicos por número de registo. Em abcissas: 1-Vegetais fósseis; 2-Foraminíferos; 3-Poríferos; 4-Corais; 5-Conulários; 6Briozoários; 7- Braquiópodes; 8 - Bivalves; 9- Gastrópodes; 10- Cefalópodes; 11Escafópodes; 12 - Rostroconchias e tentaculites; 13 - Crinóides; 14 - Blastóides, cistóides e asterozoos; 15 - Equinídeos; 16 - Anelídeos; 17- Trilobites; 18Crustáceos; 19- Insectos; 20- Protocordados; 21- Vertebrados. Em ordenadas: número de items.

Quanto à repartição estratigráfica e idades relativas representadas, estão presentes quase todos os períodos e épocas do Eon Fanerozóico, com excepção do Ordovícico e do Paleocénico (Figura 8). Não obstante, há que 
ter em conta o carácter bastante mais tardio da definição e reconhecimento do Período Ordovícico na estratigrafia clássica europeia, pelo que a indicação de Silúrico em parte dos rótulos analisados corresponderá, na realidade, a uma idade ordovícica dos fósseis e jazidas em causa. É interessante notar, também, a prevalência de amostras com espécies de idade triássica e jurássica, facto que se poderá explicar por se estar na presença de um comptoir austríaco, mais vocacionado para recolhas e trocas dentro do espaço germanófilo, onde estes andares se encontram bem representados. Independentemente destas valências, parece-nos indubitável que a colecção em si providencia a um observador não especializado, uma visão bastante precisa do universo dos fósseis estratigráficos e da biodiversidade dos últimos 500 milhões de anos.

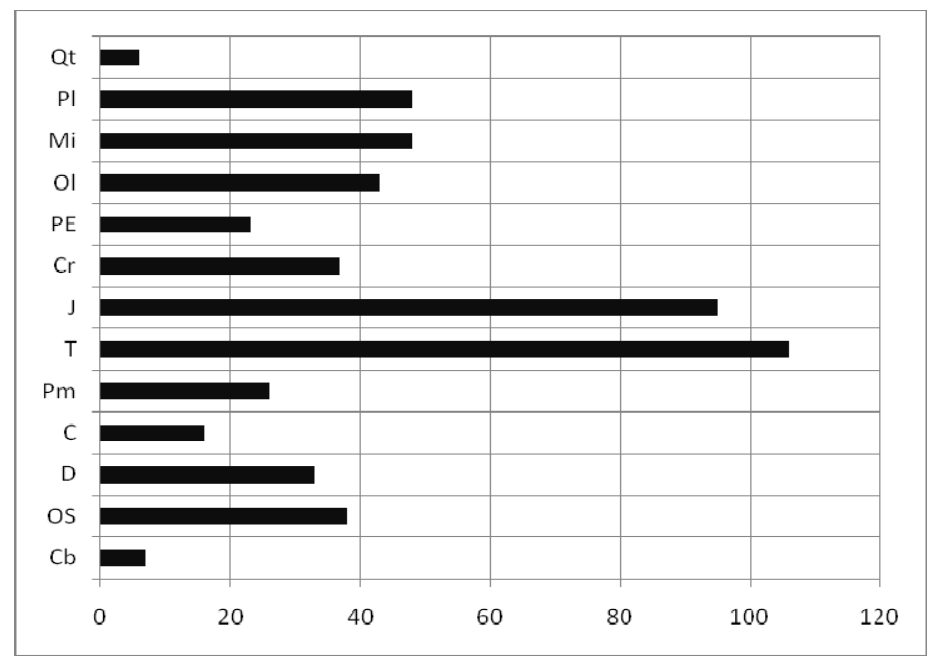

Figura 8. Representatividade relativa por número de registo dos diferentes Períodos e Épocas da escala cronoestratigráfica (Éon Fanerozóico). Cb- Câmbrico; S- Ordovícico e Silúrico; D- Devónico; C - Carbonífero; Pm- Pérmico; T- Triásico; J- Jurássico; CrCretácico; PE - Paleocénico e Eocénico; OI - Oligocénico; Mi - Miocénico; PI Pliocénico; Qt - Quaternário (Plistocénico e Holocénico).

Um último ponto forte da colecção em estudo reside na sua diversidade geográfica. Não obstante a maioria das amostras ser de proveniência europeia, existem outras de origem norte-americana ou norteafricana, estas últimas de provável procedência francesa (Figura 9). O número de países ou possessões representadas nos locais de recolha é de 17, sendo que mais de $50 \%$ dos espécimes provém de jazidas da Alemanha, França e Reino Unido. Seguem-se, ainda com frequência relativa considerável, a Itália, a República Checa, a Áustria, os Estados Unidos da 
América e a Suécia. Em proporções bem mais reduzidas estão ainda presentes diversos países europeus, incluindo a Bélgica, a Polónia, a Suíça e a Rússia. Esta diversidade apreciável reflecte o bom conhecimento que já existia, à época, da estratigrafia europeia, sendo que a Alemanha, França e Reino Unido eram, muito naturalmente, os berços da Geohistória e, como tal, os países com maior concentração de jazidas clássicas, profusamente descritas e exploradas pelas respectivas écoles.

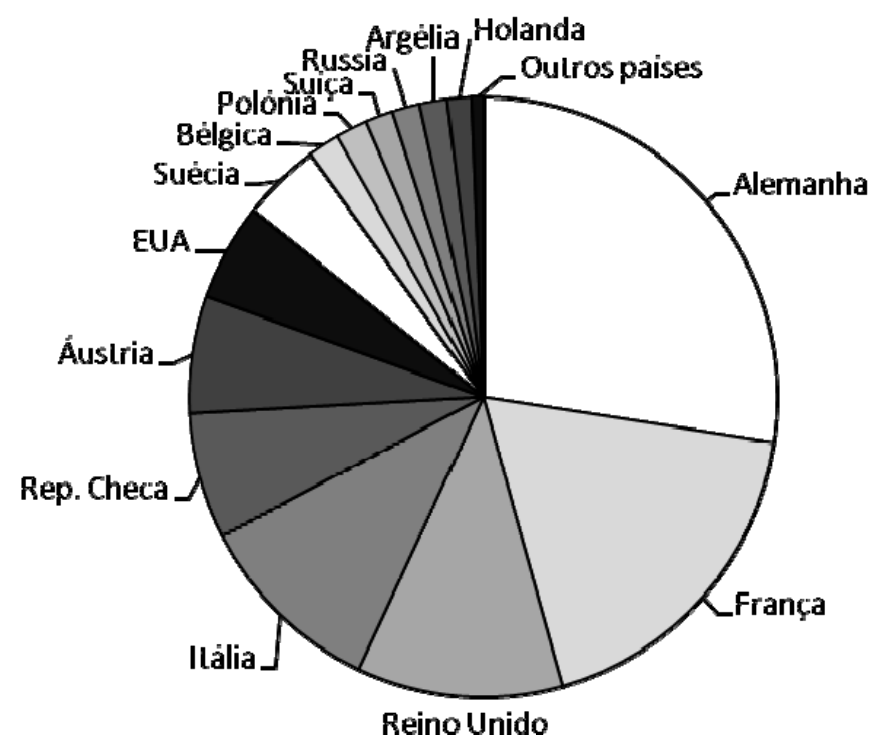

Figura 9. Representatividade relativa por número de registo dos diferentes países referentes aos locais de recolha da colecção Lenoir \& Forster.

No extremo oposto é interessante constatar a ausência total de espécimes dos países ibéricos, numa época em que a Comissão Geológica de Portugal já havia dado a conhecer muito do que existe na Estratigrafia e Paleontologia portuguesas. Esta lacuna poderá ser entendida, ainda que em parte, por um completo afastamento do país dos circuitos comerciais e de coleccionismo de fósseis, ao tempo já tão em voga noutros países da Europa ocidental. $\mathrm{Na}$ realidade, a grande diversidade geográfica subjacente à colecção constitui um excelente testemunho da extraordinária rede de trocas a que o comptoir de Lenoir \& Forster teria acesso, capaz de suprir estabelecimentos de ensino e colecções particulares, mas também de enriquecer os acervos dos grandes museus de História Natural da época, ao exemplo do que Mary Anning já fazia quase um século antes, quando em Portugal ainda se filosofava nas entrelinhas das épocas de Buffon e dos sistemas de Werner. 
Agradecimentos: Os autores homenageiam através deste trabalho o Prof. Manuel Maria Godinho, recordando vivências e ensinamentos, assim como o contributo precioso que o Cientista infatigável deu ao progresso do conhecimento geológico. Estão, também, gratos à Dra. Aurora Deshauteurs, curadora da imagoteca da Biblioteca de Filadélfia, pela gentileza de autorizar a reprodução da imagem da Figura 1.

\section{Bibliografia}

Antunes, M. T. (1986) - Sobre a história da paleontologia em Portugal. História e Desenvolvimento da Ciência em Portugal, vol. 2. Publicações do II centenário da Academia das Ciências de Lisboa, 773-814.

Areia, M. L. R. (2007) - Bernardino Machado, Professor de Antropologia. In: Machado, Bernardino. Obras: Ciência (1875-1907). Ribeirão, Edições Húmus, Lda, 39-49.

Areia, M. L. R \& Rocha, M. A. (1985) - O ensino da Antropologia. In: Cem anos de Antropologia em Coimbra 1885-1985. Coimbra, Museu e Laboratório Antropológico, 13-60.

Areia, M. L. R.; Rocha, M. A \& Miranda, M. A. (1991) - O Museu e Laboratório Antropológico da Universidade de Coimbra. In: Universidade(s). História. Memória. Perspectivas. Actas do Congresso "História da Universidade" (no $7^{\circ}$ Centenário da sua Fundação, 1990). Coimbra, Comissão Organizadora do Congresso "História da Universidade", 1991, 2, 87-105.

Bastos, H. Teixeira (1889) - Faculdade de Philosofia: Programa da $8^{\text {a }}$ cadeira Anthropologia e Archeologia Prehistorica para o anno lectivo de 1889 a 1890. Imprensa da Universidade, Coimbra, $9 \mathrm{p}$.

Ferreira, M. R. P. (1998) - 200 anos de Mineralogia e Arte de Minas: desde a Faculdade de Filosofia (1772) até à Faculdade de Ciências e Tecnologia (1972) - FCTUC, Gráfica de Coimbra Ltda., Coimbra, $188 \mathrm{p}$.

Fundo Documental: Universidade de Coimbra. SR: Documentos de despesa. Arquivo da Universidade de Coimbra.

Machado, B. (1904/1905) - A Universidade e a Nação. Oração inaugural do ano lectivo 1904-1905, em 16-10-1904. Anuário da Universidade, p. XXXIV.

Pires, N. P. (2009) - A colecção de Arqueologia do Museu Geológico. Tese de Mestrado não publicada. Faculdade de Ciências Sociais e Humanas da Universidade Nova de Lisboa, Lisboa, $117 \mathrm{p}$.

Rocha, M. A. (1995) - Les collections ostéologiques humaines identifiées du Musée Anthropologique de l'Université de Coimbra. Antropologia Portuguesa; 13, 7-38.

Rosa, E. M. T. D. M. (2006) - Bernardino Machado [Fotobiografia]. Museu da Presidência da República, Lisboa, $120 \mathrm{p}$.

Sueiro, M. A. Barbosa (1944) - Doutor Bernardino Machado (1851-1944). Imprensa Médica; ano X (9), 157-158.

http://libwww.library.phila.gov/CenCol/Details.cfm?ItemNo=c021205\&sourceURL=cedcpubsrch9.cf m. [em 20.11.2010] 


\section{Anexos}

Anexo 1. Elenco taxonómico da colecção de Paleontologia adquirida ao comptoir de Lenoir \& Forster.

Fósseis vegetais: Acer trilobatum, Dalbergia bella, Sapindus faleifolius, Populus mutabilis, Acer trilobatum, Salix angusta, Quercus elaena, Cinnamomum lanceolatum, Taxodium langsdorfii, Folliculites kaltennordnheimensis, Viburnum dakotense, Betulites vestii, Sphenolepidium kurrianum, Sphenopteris hartlebeni, Codites serpentinus, Fucoides bollensis, Nilssonia acuminata, Zamites disparis, Calamites gumbeli, Pterophyllum jaegeri, Pterophyllum pulchellum, Asterocarpus keriani, Calamites arenaceus, Voltzia heterophylla, Voltzia liebeana, Ullmannia frumentaria, Walehia piriformis, Psaronius cottae, Psaronius helmintholithus, Araucarioxylon saxonicum, Astrophylliles equidetiformis, Pecopteris unita, Annularia longifolia, Pecopteris villosa, Neuropteris hirsuta, Pecopteris arborescens, Pecopteris muricata, Todea lipoldi, Calamites approximatus, Neuropteris gigantea, Asterophyllites equiseliformis, Sphenopseris obtusiloba, Sphenopteris divariesta, Sphenopteris fureata, Lonchopteris rugosa, Polytomella crispa, Lithothamnium pliocenicum

Foraminíferos: Bulimia echinata, Quinqueloculina rugosa, Vaginalina legumen, Globulina gibba, Orbitoides nummulitica, Nummulites mammilata, Nummulites laevigatus, Siderolites calcitrapoides, Orbitolites socialis, Orbitolina concava, Fusulina cylindrica

Espongiários: Coscinopora infundibuliformis, Coeloptychium decimium, Jereica pyriformis, Pachytilodia infundibuliformis, Stauronema carteri, Plocoscyphia fenestrata, Raphidonema faringdonense, Cnemidiastrum goldfussi, Hyalofragos patella, Cypellia rugosa, Tremadictyon reticulatum, Stellispongia glomerata, Corynella quenstedti

Corais: Cariophyllia clavus, Flabellum avicula, Oculina crassoramosa, Trochocyatus versicostatus, Balanophyllia sinuata, Plocophyllia flabellata, Heliastraea lucasana, Phyllocoenia irradians, Dendrophyllia cariosa, Diplohelia raristella, Crochocyathus allonensis, Trochosmilia cernua, Columnastraea seriata, Agathelia asperella, Thamnastraea procera, Isastraea profunda, Cyclolites macrostoma, Lophomillia cenomana, Cyclolites spinosa, Aspidiscus cristatus, Cyclocyathus fittoni, Stylina limbata, Montlivaltia obconicum, Discocyathus euclesi, Michelinia favosa, Lonsdaleia floriformis, Cyathophyllum helianthoides, Cyathophyllum quadrigeminum, Cystiphyllum lamellosum, Calceola sandalina, Favosites polymorpha, Heliolites porosa, Aulopora repeus, Actinostroma clathratum, Pleurodiction problematicum, Labechia conferta, Heliolites inlerstinctus, Halysites catenularia

Conulariídeos: Conularia cristata

Briozoários: Cellepora tubigera, Myriozoum truncatum, Cupularia umbellata, Lepralia granulifera, Eschara fascialis, Retecava clathrata, Periopora ligeriensis, Eschara sexangularis, Plethopora pseudotroquata, Sparsicavea dichotoma, Ceriopora polymorpha, Ceriopora polymorpha, Ceriopora radiciformis, Acanthocladia anceps, Stenopora columnaris, Chaetetes radians, Monticulipora tumida, Monticulipora fibrosa, Fenestella nobilis, Stellipora autheloidea

Braquiópodes: Terebratula ampula, Terebratula sinuosa, Terebratula grandis, Rhynchonella polymorpha, Terebratula obesa, Terebratulina chrysalis, Trigonosemus pectiniformis, Trigonosemus pectitum, Rhynchonella plicatilis, Crania antigua, Thecidium hieroglyphicum, Megerlea puslulosa, Rhynchonella cuvieri, Terebratula semiglobosa, Terebratula Nerviensis, Terebratulina biauriculata, Terebratula sella, Rhynchonella depressa, Terebratula diphya, Terebratula bisufforcinata, Terebratula insignis, Waldheimia impressa, Rhynchonella caenuosa, Rhynchonella spathica, Megerlea pectunculoides, Waldheimia perovalis, Terebratula sphaeroidalis, Rhynchonella tetraedra, Rhynchonella mimosa, Terebratula subpunctata, Waldheimia resupinata, Waldheimia numismalis, Terebratula pyriformis, Terebratula gregaria, Athyris orycolpos, Koninkina leonbardi, Terebratula angusta, Waldheimia vulgaris, Spiriferina fragilis, Spirifer alata, Strophalosia 
goldfussi, Productus horridus, Spirifer mosquensis, Athyris roysii, Productus pustulosus, Productus semireticulatus, Camarophoria formosa, Stringocephalus burtini, Uncites gryphus, Orthis strialula, Atrypa reticulata, Streplorhynchus umbraculum, Anoplotheca lepida, Retzia ferita, Cyrlina heteroclyta, Spirifer curvatus, Spirifer laevicosta, Spirifer cultrijugatus, Chonetes tenuicostata, Orthis vulvaria, Rensselaeria strigiceps, Strophomena laticosta, Spirifer macropterus, Rhynchonella livonica, Rhynchonella princeps, Pentamerus acutilobatus, Daya navicula, Strophomena euglypha, Leptaena depressa, Spirifera sulcata, Nucleospira pisum, Strioklandinia lirata, Orthis redrus, Lophonema deltoidea, Lingula darisi

Moluscos bivalves: Pholas crispata, Saxicava arthica, Mya truncata, Sphaerium solidum, Pisidium amnicum, Pecten Jacobeus, Limopsis auritus, Venus senilis, Astarte fusca, Chama gryphoides, Mactra triangularis, Cytherea affinis, Arca diluvii, Lucina columbella, Crassatella dissita, Pectunculus obovatus, Cyprina aequalis, Perna soldani, Mytilus aquitanicus, Cyrena semistriata, Cardita planicosta, Cardium purulosum, Cytherea semisulcata, Corbis lamellosa, Ostrea bellovacina, Ostrea nicaisei, Ostrea ungulata, Vola quadricostata, Spondylus dutemplei, Cyrena garumnica, Crassatella macrodonta, Radiolites toucasi, Hippurites Toucasi, Hippurites cornuvaccinum, Ostrea hippopodium, Ostrea Matheroniana, Lima texta, Cyprina planata, Pecten acuminatus, Inoceramus sulcatus, Exogyra couloni, Cyrena fasciata, Lucina substriata, Cyrena nuculaeformis, Thracia incerta, Pholadomya decussata, Modiola sowerbyi, Panopaea jurassi, Astarte obliqua, Trigonia costata, Myoconcha liasica, Gryphaea arcuata, Lima gigantea, Avicula contorta, Daonella lomelli, Corbula rosthomi, Myophoria keffersteini, Avicula ?gaea, Megalodon carinthiacum, Gervillia socialis, Lima striata, Myopheria simplex, Avicula speluncaria, Gervillia ceratophaga, Nucula ventricosa, Posidonomya becheri, Cardiola retrostriata, Lucina proavia, Venulites concentricus, Pterinea lineata, Palaeosolen costatus, Valhila explanata, Pterinea retroflexa, Modiolopsis morieri, Corbula infundibulum

Moluscos gastrópodes: Trophon clathratus, Buccinum nivosum, Lymnaea palustris, Hyalina cellaria, Murex polymorpha, Fusus longirostris, Nassa clathrata, Triton affine, Cassis saburon, Ranella marginata, Cancellaria lyrata, Columbella nassoides, Mitra scrobiculata, Vermetus intortus, Cypraea sanguinolenta, Ancillaria glandiformis, Conus fuscocingulatus, Turritella triplicata, Terebra pescata, Pleurotoma dimidiata, Cerithium bronni, Natica millepunctata, Paludina naricosa, Helix deflexa, Pleurotoma lelysii, Aporrhais tridactylus, Voluta rathieri, Cerithium plicatum, Rostellaria fissurella, Fusus bulbiformis, Voluta cytherea, Rostellaria columbaria, Cassidaria nodosa, Turritella imbricarária, Turritella coquandi, Actaeonella conica, Cerithium simonyi, Glauconia conoidea, Nerinaea gracilis, Pleurotomaria neptuni, Solarium ornatum, Rostellaria carinata, Nerinea schiosensis, Paludina fluviorum, Pteroceras oceani, Natica turbiniformis, Chemnitzia herringlonensis, Trochus subduplicatus, Pleurotomaria conoidea, Alaria rouboleti, Pleurotomaria amalthei, Chemnitzia aldrovandi, Turbo depressus, Chemnitzia maioroni, Pleurotomaria radians, Chemnitzia alpina, Holopella glacilior, Natica minima, Bellerophon sublaevis, Platyceras aequilateralis, Ampullaria helicoides, Euomphalus latus, Macrocheilus arculatus, Pleurotomaria daleydensis, Murchisonia verneuili, Capulus priscus, Capulus hercynicus, Eulima spinosa, Platyceras cornutum, Euomphalus discors

Moluscos cefalópodes: Baculites ovalis, Scaphites nodosus, Acanthoceras rhotomagensis, Scaphites aequalis, Turrilites costatus, Ammonites interruptus, Ammonites auritus, Hamites attenuatus, Belemnites minimus, Nautilus inaequalis, Ammonites dufrenoyi, Ammonites guettardi, Ammonites deshayesi, Ammonites infundibulum, Crioceras villiersianus, Belemnites dilatatus, Reineckia reineckiana, Phylloceras plychoicum, Perisphinctes polygyratus, Oppelia flexuosa, Aspidoceras perarmatus, Perisphinctes plicatilis, Belemnites giganteus, Peltoceras annularis, Macrocephalites macrocephalus, Parkinsonia parkinsoni, Stephanoceras braikenridgei, Sphaeroceras wrightii, Harpoceras murchisonae, Harpoceras opalinus, Belemnites papillosus, Ammonites nilssoni, Harpoceras radius, Harpoceras bifrons, Amaltheus margaritatus, Aegoceras pettos, Aegoceras raricostatus, Arietites conybeari, Arietites roliformis, Arcestes boeckhi, Arcestes 
intuslabiatus, Joannites cymbiformis, Cladiscites tornatus, Ceratites nodosus, Orthoceras monoceros, Goniatites oweni, Goniatites sphaericus, Clymenia nudulata, Goniatites retrorsus, Goniatites intumescens, Gyroceras nodosum, Orthoceras crelnum, Orthoceras angulatum, Cyrtoceras thetidis, Goniatites plebeius, Hercoceras mirum

Moluscos escafópodes: Denthalium sexangulum, Dentalium mosae, Dentalium laeve

Outros moluscos: Conocardium arabicum, Tentaculites scalaris

Crinóides: Marsupites ornatus, Eugeniacrinites caryophyllata, Pentacrinus tuberculatus, Apiocrinus mespiliformis, Encrinus lilliformis, Cyathocrinus ramosus, Moscovicrinus multiplex, Batocrinus rotundus, Platycrinus hemisphaericus, Rhodocrinus crenatus, Symbathocrinus tabulatus, Cupressocrinus elongatus, Cyathocrinus goniodactylus

Blastóides e Cistóides: Pentatrematites florealis, Echinosphaerites aurantium

Asterozoos e Ofiuróides: Asterias lumbricalis, Ophiura arenacea, Furcaster palaeozoicus, Roemeraster asperula

Equinóides: Eihinolampas kleinii, Conoclypus conoideus, Eohinolampas affinis, Cyphosoma delamarrei, Heterodiadema lybicum, Orthopsis miliaris, Salenia bourgeoisi, Holectypus serialis, Galerites albogalerus, Anachytes ovata, Heterolampas maresi, Calopygus fenestratus, Hemiaster fourneli, Micraster coranguinum, Echinobrissus subsetifensis, Epiaster meridaneus, Holaster subglobosus, Discoidea forgemolli, Holaster laevis, Echinospatangus ricordianus, Heteraster fissoli, Peltastes wrightii, Melaporhinus convexus, Cidaris coronata, Stomechinus bigranularis, Collyrites ringens, Clypeus albus, Pygaster semisulcatus, Eocidaris keyserlingi, Archaeocidaris rossica

Anelídeos: Serpula tuba, Serpula lumbricalis, Serpula gordialis, Spirorbis ammonia

Trilobites: Phillipsia longicornis, Cryphaeus punctatus, Phacops latifrons, Phacops ferdinandi, Cryphaeus laciniatus, Beyrichia bohemica, Bronteus umbellifer, Cheirurus stembergi, Dalmanites caudatus, Proetus elegantulus, Sphaeroxochus mirus, Acidaspis mira, Lichas palmata, Cromus beaumonti, Harpes ungula, Dalmanites phillipsi, Trinucleus granatus, Nileus armadillo, Asaphus expansus, Asaphellus humphreyi, Ellipsocephalus hoffi, Arionellus ceticephalus, Agnostus nathorsti

Crustáceos: Beyrichia bohemica, Carcinus wurmlembergicus, Glyphthyreus wetherelli, Lanthopsis leachi, Lanthopsis kressenbergensis, Hoploparia longimana, Meyeria vectensis, Penreus speciosus, Eryma modesti formis, Acanthocinus augustus, Eryon schuberti, Mecochirus longimanus, Eryon arctiformis, Balanus tintinnabulum, Cypris valdensis, Leperdilia baltica, Aristozoe regina

Insectos: Acanthocinus augustus, Sitona atavina, Diptera [asa], Stenophlebia aequalis

Protocordados: Monoprion priodon

Vertebrados: Otodus appendiculatus, Clupea brevissima, Leptolepis sprattiformis, Palaeoniscus freieslebenensis, Psammodus porosus, Osteolepis microlepidotus, Protiton petrolei, [pena de ave], Hyaena spelaea, Aceratherium (Rhinoceros) incisivum, Prolagus sardus, Oreodon culbertsoni, Caenotherium leptognathum, Palaeotherium curtum

Icnofósseis: Spirophyton eifeliense, Planulites ?jameshalli 